\title{
Appendix A: Notations
}

Table A1. Notations

\begin{tabular}{|c|c|c|}
\hline Symbols & Units & Definitions \\
\hline$*$ & - & represents the mathematical convolution function \\
\hline$C_{M}$ & {$\left[\mathrm{~m} \cdot \mathrm{s}^{-1}\right]$} & parameter controlling the solute mass flux celerity \\
\hline$C_{Q}$ & {$\left[\mathrm{~m} . \mathrm{s}^{-1}\right]$} & flood wave celerity \\
\hline$D_{M}$ & {$\left[\mathrm{~m}^{2} \cdot \mathrm{s}^{-1}\right]$} & parameter controlling the solute mass flux diffusivity \\
\hline$D_{Q}$ & {$\left[\mathrm{~m}^{2} \cdot \mathrm{s}^{-1}\right]$ flood wave diffusivity } & \\
\hline$\left(K_{Q}, K_{M}\right)$ & - & Hayami kernel function for water flow and mass flux modelling, respectively \\
\hline$m$ & {$\left[\mathrm{~kg} \cdot \mathrm{s}^{-1} \cdot \mathrm{m}^{-1}\right]$} & lateral mass flux per length unit \\
\hline$M$ & {$\left[\mathrm{~kg} \cdot \mathrm{s}^{-1}\right]$} & mass flux \\
\hline$\left(M_{I, \text { base }}, M_{I, \text { flood }}, M_{I, \text { tot }}\right)$ & {$\left[\mathrm{kg} \cdot \mathrm{s}^{-1}\right]$} & upstream base, flood and total mass flux, respectively \\
\hline$\left(M_{O, \text { base }}, M_{O, \text { flood }}, M_{O, \text { tot }}\right)$ & {$\left[\mathrm{kg} \cdot \mathrm{s}^{-1}\right]$} & downstream base, flood and total mass flux, respectively \\
\hline$\left(M_{A, \text { base }}, M_{A, \text { flood }}, M_{A, \text { tot }}\right)$ & {$\left[\mathrm{kg} \cdot \mathrm{s}^{-1}\right]$} & lateral base, flood and total mass flux exchanges, respectively \\
\hline$q$ & {$\left[\mathrm{~m}^{2} \cdot \mathrm{s}^{-1}\right]$} & lateral flow per length unit \\
\hline$Q$ & {$\left[\mathrm{~m}^{3} \cdot \mathrm{s}^{-1}\right]$} & discharge \\
\hline$\left(Q_{I, \text { base }}, Q_{I, \text { flood }}, Q_{I, \text { tot }}\right)$ & {$\left[\mathrm{m}^{3} \cdot \mathrm{s}^{-1}\right]$} & upstream base, flood and total flow, respectively \\
\hline$\left(Q_{O, \text { base }}, Q_{O, \text { flood }}, Q_{O, \text { tot }}\right)$ & {$\left[\mathrm{m}^{3} \cdot \mathrm{s}^{-1}\right]$} & downstream base, flood and total flow, respectively \\
\hline$\left(Q_{A, \text { base }}, Q_{A, \text { flood }}, Q_{A, t o t}\right)$ & {$\left[\mathrm{m}^{3} \cdot \mathrm{s}^{-1}\right]$} & lateral base, flood and total flow exchanges, respectively \\
\hline$(R 1, R 2)$ & - & reach 1 (s1 to s2) and reach 2 (s2 to $\mathrm{s} 3)$, respectively \\
\hline$(s 1, s 2, s 3)$ & - & monitoring station 1,2 and 3 , respectively \\
\hline$\left(S_{I, \text { base }}, S_{I, \text { flood }}, S_{I, \text { tot }}\right)$ & {$\left[\mathrm{g} . \mathrm{l}^{-1}\right]$} & upstream solute base, flood and total concentrations, respectively \\
\hline$\left(S_{O, \text { base }}, S_{O, \text { flood }}, S_{O, \text { tot }}\right)$ & {$\left[\mathrm{g} . \mathrm{l}^{-1}\right]$} & downstream solute base, flood and total concentrations, respectively \\
\hline$\left(S_{A, \text { base }}, S_{A, \text { flood }}, S_{A, \text { tot }}\right)$ & {$\left[\mathrm{g} . \mathrm{l}^{-1}\right]$} & lateral solute base, flood and total concentrations, respectively \\
\hline$t$ & {$[\mathrm{~s}]$} & time \\
\hline$U$ & - & percentage of the unsaturated conduit length along R2 \\
\hline$x$ & {$[\mathrm{~m}]$} & downstream distance \\
\hline
\end{tabular}

